TRANSACTIONS OF THE

AMERICAN MATHEMATICAL SOCIETY

Volume 357, Number 6, Pages 2483-2507

S 0002-9947(04)03617-7

Article electronically published on November 23, 2004

\title{
CLUSTERING IN COAGULATION-FRAGMENTATION PROCESSES, RANDOM COMBINATORIAL STRUCTURES AND ADDITIVE NUMBER SYSTEMS: ASYMPTOTIC FORMULAE AND LIMITING LAWS
}

\author{
GREGORY A. FREIMAN AND BORIS L. GRANOVSKY
}

\begin{abstract}
We develop a unified approach to the problem of clustering in the three different fields of applications indicated in the title of the paper, in the case when the parametric function of the models is regularly varying with positive exponent. The approach is based on Khintchine's probabilistic method that grew out of the Darwin-Fowler method in statistical physics. Our main result is the derivation of asymptotic formulae for the distribution of the largest and the smallest clusters (= components), as the total size of a structure (= number of particles) goes to infinity. We discover that $n^{\frac{1}{l+1}}$ is the threshold for the limiting distribution of the largest cluster. As a byproduct of our study, we prove the independence of the numbers of groups of fixed sizes, as $n \rightarrow \infty$. This is in accordance with the general principle of asymptotic independence of sites in mean-field models. The latter principle is commonly accepted in statistical physics, but not rigorously proved.
\end{abstract}

\section{Introduction: The objective And the CONTEXT}

We develop a unified approach to the problem of clustering in the three different fields of applications indicated in the title of the paper. The approach is based on Khintchine's probabilistic method that grew out of the Darwin-Fowler method in statistical physics. To the best of our knowledge, the first application of Khintchine's method for coagulation-fragmentation processes was made in [20], where it was used for the derivation of asymptotic formulae for the partition function of the invariant measure of the process. The present paper extends the method to much more complicated asymptotic problems arising in the study of clustering.

Our main result is the derivation of asymptotic formulae for the distribution of the largest and the smallest clusters (= components), as the total size of a structure (= number of particles) goes to infinity.

The organization of the paper is as follows. Section 2 provides a formal mathematical setting that encompasses the clustering problems arising in the contexts of coagulation-fragmentation processes, random combinatorial structures and additive number systems. The mathematical problem is stated as follows. Let the functions $g, S: \mathbb{C} \rightarrow \mathbb{C}$ be related via $g(z)=e^{S(z)},|z|<R, R>0$. Under a given asymptotic

Received by the editors July 18, 2002 and, in revised form, January 7, 2004.

2000 Mathematics Subject Classification. Primary 60K35, 05A15; Secondary 05A16, 05C80, $11 \mathrm{M} 45$.

Key words and phrases. Coagulation-fragmentation process, random combinatorial structures, local limit theorem, distributions on the set of partitions, additive number systems. 
behavior of the Taylor coefficients $\left\{a_{n}\right\}$ of the function $S$ one must explore the asymptotic behavior of certain quantities related to the Taylor coefficients $\left\{c_{n}\right\}$ of the function $g$.

The problem is considered for the class of functions $S$, such that $a_{n} \sim n^{l-1} L(n)$, $l>0, n \rightarrow \infty$, where $L$ is a slowly varying function. A specific feature of this class of functions is that it provides the validity of the normal local limit theorem for the associated probabilistic model.

In Section 3 we explain the idea of Khintchine's method and apply it to the derivation of the asymptotic formulae for the limiting distributions of the largest and the smallest clusters. We find that $n^{\frac{1}{l+1}}$ is the threshold for the limiting distribution of the largest cluster.

In Sections 4-6 we demonstrate how to interpret these asymptotic formulae in the context of the aforementioned three fields, and we provide a description of the striking picture of clustering that follows from these formulae. It turns out that for large $n$, almost all weight of $n$ is distributed into groups of sizes about $n^{\frac{1}{l+1}}$, while the rest of the weight is made up of groups of small sizes.

As a by-product of our study:

(i) We prove the independence of the numbers of groups of fixed sizes, as $n \rightarrow \infty$. This is in accordance with the general principle of asymptotic independence of sites in mean-field models. The latter principle is commonly accepted in statistical physics, but not rigorously proved.

(ii) We recover an asymptotic result by J. Knopfmacher, A. Knopfmacher and R. Warlimont, that is widely known in the theory of additive number systems.

\section{Mathematical Setting And PReliminaries}

We consider throughout the paper the set $\mathcal{F}(l), l>0$, of sequences $a=\left\{a_{n}\right\}_{1}^{\infty}$, $a_{n} \geq 0, n \geq 1$, with the following asymptotic behavior:

$$
a_{n} \sim n^{l-1} L(n), \quad \text { as } n \rightarrow \infty, \quad l>0 .
$$

Here and in what follows, $L$ is a slowly varying (s.v.) function at infinity (for references see [10, [37). We will need the following two asymptotic properties of s.v. functions:

$$
\begin{gathered}
L(x)=o\left(x^{\epsilon}\right), \quad \text { as } x \rightarrow \infty, \quad \text { for all } \epsilon>0, \\
x^{-\epsilon}=o(L(x)), \quad \text { as } x \rightarrow \infty, \quad \text { for all } \epsilon>0 .
\end{gathered}
$$

We assume further that

- $L$ is differentiable on $[0, \infty)$. This is based on the fact ([37, p. 17) that for any s.v. function $L$ there exists a s.v. function $\tilde{L}$, that possesses the aforementioned property and satisfies $L(x) \sim \tilde{L}(x)$, as $x \rightarrow \infty$.

- The function $x^{-\delta} L(x)$ is locally bounded on $[0, \infty)$, for any $\delta>0$.

It is easy to derive from the representation of the set of s.v. functions ([37], p. 2) that the sequences $a \in \mathcal{F}(l), l>0$, satisfy

$$
\lim _{n \rightarrow \infty} \frac{a_{n}}{a_{n+1}}=1 \text {. }
$$

We will also need the fact that a s.v. function $L$ has a conjugate function $L^{*}$ ([37], p. 25 and [10], p. 47), which is also a s.v. function and is uniquely defined (up to 
asymptotic equivalence) by the asymptotic relationship

$$
L^{*}(x) L\left(x L^{*}(x)\right) \sim L(x) L^{*}(x L(x)) \sim 1, \quad \text { as } \quad x \rightarrow \infty .
$$

(2.5) says that the asymptotic behavior of $L^{*}$ is converse to the one of $L$, in the sense that

$$
\lim _{n \rightarrow \infty} L^{*}(n)=\left(\lim _{n \rightarrow \infty} L(n)\right)^{-1},
$$

provided the limits exist.

The characterization of the class of sequences $\mathcal{F}(l), l>0$, is given by the celebrated Karamata Tauberian theorem (for references see [37, p. 59 and [18], p. 423) that is a widely used tool in different fields of probability.

In effect, we will employ the following corollary of Karamata's theorem.

Theorem 1 (18], p. 423). Let a sequence $a=\left\{a_{n} \geq 0, n \geq 1\right\}$ be ultimately monotone, and suppose that the radius of convergence of the power series (in $z$ )

$$
S(z)=\sum_{n=1}^{\infty} a_{n} z^{n}, \quad z \in \mathbb{C},
$$

equals 1. Then the two conditions (i) and (ii) are equivalent:

$$
S(z) \sim \frac{\Gamma(l)}{(1-z)^{l}} L\left(\frac{1}{1-z}\right), \quad l>0, \quad \text { as } z \rightarrow 1^{-},
$$

where $\Gamma$ is the gamma function, and

$$
a_{n} \sim n^{l-1} L(n) \in \mathcal{F}(l), \quad l>0 .
$$

For our subsequent study we will make use only of the abelian part (i) of the above theorem.

Next we define the sequence $c=\left\{c_{n}\right\}_{0}^{\infty}$ generated by the above sequence $a$ in the following manner:

$$
g(z):=\sum_{n=0}^{\infty} c_{n} z^{n}=e^{S(z)}, \quad|z|<1 .
$$

We will demonstrate in Sections 4-6 that the above form of the exponential relationship between two generating functions arises in the three fields in the title of the present paper. In view of this, a variety of problems related to (2.10) (but quite different from the problem considered by us) have been studied by many researchers.

Based on (2.4), it is easy to derive (see [13] and [11], Lemma 1.22) that the radius of convergence of the series for $g(z)$ equals 1 . Moreover, it was recently proven by J. Bell and S. Burris (8], Lemma 4.2) that (2.4) implies

$$
\lim _{n \rightarrow \infty} \frac{c_{n}}{c_{n+1}}=1 .
$$

This fact is important, since by Compton's density theorem (see for references 8 and [11], Ch. 4), the condition (2.11) implies that all partition sets of an additive number system have asymptotic density which is either 0 or 1 . 
To formulate the problem of clustering that is addressed in the present paper we introduce some more notation. For given $r, n, 1 \leq r=r(n) \leq n, n=1,2, \ldots$, we denote

$$
\underline{S}_{n}^{(r)}(z)=\sum_{j=1}^{r} a_{j} z^{j}, \quad \bar{S}_{n}^{(r)}(z)=\sum_{j=r}^{n} a_{j} z^{j}, \quad|z|<1,
$$

and consider the two power series

$$
\underline{g}_{n}^{(r)}(z)=e^{\underline{S}_{n}^{(r)}(z)}:=\sum_{j=0}^{\infty} \underline{c}_{j}^{(r)} z^{j} \quad \text { and } \quad \bar{g}_{n}^{(r)}(z)=e^{\bar{S}_{n}^{(r)}(z)}:=\sum_{j=0}^{\infty} \bar{c}_{j}^{(r)} z^{j}, \quad|z|<1 .
$$

Setting $r=n^{\beta}, 0 \leq \beta<1$ and denoting $\underline{c}_{n}^{(n)}=c_{n}, n=1,2, \ldots$, our ultimate objective will be the derivation of the limits, as $n \rightarrow \infty$, for the two quantities

$$
\underline{d}_{n}^{(r)}:=\frac{\underline{c}_{n}^{(r)}}{c_{n}} \quad \text { and } \quad \bar{d}_{n}^{(r)}:=\frac{\bar{c}_{n}^{(r)}}{c_{n}} .
$$

Here and in what follows we agree that $r=\bullet$ means that $r=[\bullet]$, where $[\bullet]$ is the integer part of the number $\bullet$.

\section{Asymptotic Formulae And Limiting LAWs}

We will study the above posed problem with the help of the probabilistic method formulated by Khintchine in [29, Chs. IV, V (see also 20]). Independently of the context of the problem considered, the implementation of Khintchine's method for deriving asymptotic formulae always follows the following two-step scheme:

(i) The construction of an auxiliary probabilistic model with a free parameter that enables one to express the quantity in question via the probability function of a sum of independent integer-valued random variables forming a triangular array.

(ii) The proof of the normal local limit theorem via a proper choice of a free parameter in the probabilistic model in (i).

The problem formulated in (2.14) requires the derivation of asymptotic formulae for the coefficients $\underline{c}_{n}^{(r)}$ and $\bar{c}_{n}^{(r)}$, for all $r=n^{\beta}, 0 \leq \beta<1$. In the case $L(x) \equiv 1$ such a formula for $\underline{c}_{n}^{(n)}$ was established in [20], with the help of Khintchine's method. Our primary aim in this section will be to extend the method to the aforementioned problem (2.14). This will require a much more complicated asymptotic analysis.

The probabilistic model suggested below is a modification of the one in [20. We start by setting in (2.12), (2.13)

$$
z=e^{-\sigma+2 \pi i \alpha},
$$

for some $\sigma, \alpha \in R$. Then, analogous to Lemma 1 in [20], the following representations of $\underline{c}_{n}^{(r)}$ and $\bar{c}_{n}^{(r)}$ are valid:

$$
\begin{gathered}
\underline{c}_{n}^{(r)}=e^{n \sigma} \int_{0}^{1} \prod_{j=1}^{r}\left(\sum_{k=0}^{\infty} \frac{a_{j}^{k} e^{-j k \sigma+2 \pi i \alpha j k}}{k !}\right) \times e^{-2 \pi i \alpha n} d \alpha, \\
\bar{c}_{n}^{(r)}=e^{n \sigma} \int_{0}^{1} \prod_{j=r}^{n}\left(\sum_{k=0}^{\infty} \frac{a_{j}^{k} e^{-j k \sigma+2 \pi i \alpha j k}}{k !}\right) \times e^{-2 \pi i \alpha n} d \alpha, \\
1 \leq r \leq n, \quad n=1,2, \ldots,
\end{gathered}
$$


where $\sigma \in R$ is arbitrary. For this reason $\sigma$ is called a free parameter. It plays an important role in the method.

To attribute a probabilistic meaning to the RHS's in (3.2), we make use of the following notation:

$$
\begin{gathered}
p_{j k}=\frac{\left(a_{j} e^{-\sigma j}\right)^{k}}{k ! \exp \left(a_{j} e^{-\sigma j}\right)}, \quad j=1, \ldots, n, \quad k=0,1, \ldots, \\
\varphi_{j}(\alpha)=\sum_{k=0}^{\infty} p_{j k} e^{2 \pi i \alpha j k}, \quad \alpha \in R, \quad 1 \leq j \leq n, \\
\underline{\varphi}^{(r)}(\alpha)=\prod_{j=1}^{r} \varphi_{j}(\alpha), \quad \bar{\varphi}^{(r)}(\alpha)=\prod_{j=r}^{n} \varphi_{j}(\alpha), \quad \alpha \in R .
\end{gathered}
$$

Notice that for a given $j$, (3.3) can be viewed as the Poisson probability function with parameter $a_{j} e^{-\sigma j}, \sigma \in R$. Now (3.2) can be rewritten as

$$
\begin{gathered}
\underline{c}_{n}^{(r)}=\exp \left(\underline{S}_{n}^{(r)}\left(e^{-\sigma}\right)+n \sigma\right) \int_{0}^{1} \underline{\varphi}^{(r)}(\alpha) e^{-2 \pi i \alpha n} d \alpha, \\
\bar{c}_{n}^{(r)}=\exp \left(\bar{S}_{n}^{(r)}\left(e^{-\sigma}\right)+n \sigma\right) \int_{0}^{1} \bar{\varphi}^{(r)}(\alpha) e^{-2 \pi i \alpha n} d \alpha, \\
1 \leq r \leq n, \quad n=1,2, \ldots .
\end{gathered}
$$

The representations (3.6), (3.7) belong to the core of Khintchine's method. The idea behind the representations is that $\underline{\varphi}^{(r)}(\alpha)$ in (3.6) can be interpreted as a characteristic function of the sum $\underline{Y}_{n}^{(r)}=X_{1}+\ldots+X_{r}$ of independent lattice random variables $X_{1}, \ldots, X_{r}, 1 \leq r \leq n$, defined by

$$
\operatorname{Pr}\left(X_{j}=j k\right)=p_{j k}, \quad j=1, \ldots, r, \quad k=0,1, \ldots
$$

Hence,

$$
\underline{T}_{n}^{(r)}=\underline{T}_{n}^{(r)}(\sigma):=\int_{0}^{1} \underline{\varphi}^{(r)}(\alpha) e^{-2 \pi i \alpha n} d \alpha=\operatorname{Pr}\left(\underline{Y}_{n}^{(r)}=n\right) .
$$

Analogously, writing $\bar{Y}_{n}^{(r)}=X_{r}+\ldots+X_{n}, 1 \leq r \leq n$, we get

$$
\bar{T}_{n}^{(r)}=\bar{T}_{n}^{(r)}(\sigma):=\int_{0}^{1} \bar{\varphi}^{(r)}(\alpha) e^{-2 \pi i \alpha n} d \alpha=\operatorname{Pr}\left(\bar{Y}_{n}^{(r)}=n\right) .
$$

In view of (3.6), (3.7) and (3.9), (3.10), we will focus now on finding the asymptotic behavior of the probabilities $P\left(\underline{Y}_{n}^{(r)}=n\right)$ and $P\left(\bar{Y}_{n}^{(r)}=n\right)$, as $n \rightarrow \infty$.

First recall that the classical normal local limit theorems (see [23], 36], p. 78, [26]) are restricted to the case of a sum of independent lattice random variables, while in our case, as we will see later on, the lattice random variables $X_{j}$ given by (3.8) with $\sigma$ depending on $n$ form a triangular array. So, even the existence of the limiting probability density for our problem is in question.

Notwithstanding this, we will demonstrate (Theorem 1 below) that a proper choice of the free parameter $\sigma$ guarantees a version of the famous Gnedenko local limit theorem.

Let $\underline{M}_{n}^{(r)}=\underline{M}_{n}^{(r)}(\sigma):=E \underline{Y}_{n}^{(r)}, \quad\left(\underline{B}_{n}^{(r)}\right)^{2}=\left(\underline{B}_{n}^{(r)}\right)^{2}(\sigma):=\operatorname{Var} \underline{Y}_{n}^{(r)}$ and $\underline{\rho}_{n}^{(r)}=$ $\underline{\rho}_{n}^{(r)}(\sigma):=E\left(Y_{n}^{(r)}-E Y_{n}^{(r)}\right)^{3}$, and denote by $\bar{M}_{n}^{(r)}=\bar{M}_{n}^{(r)}(\sigma),\left(\bar{B}_{n}^{(r)}\right)^{2}=\left(\bar{B}_{n}^{(\bar{r})}\right)^{2}(\sigma)$ and $\bar{\rho}_{n}^{(r)}=\bar{\rho}_{n}^{(r)}(\sigma)$ the same moments of the sum $\bar{Y}_{n}^{(r)}$. 
It follows from (3.8) that $j^{-1} X_{j}, j=1, \ldots, n$, are Poisson $\left(a_{j} e^{-\sigma j}\right)$ random variables. So, we have the following expressions for the above quantities:

$$
\begin{aligned}
& \underline{M}_{n}^{(r)}=\sum_{j=1}^{r} j a_{j} e^{-\sigma j}, \quad \bar{M}_{n}^{(r)}=\sum_{j=r}^{n} j a_{j} e^{-\sigma j}, \quad n=1,2, \ldots, \\
& \left(\underline{B}_{n}^{(r)}\right)^{2}=\sum_{j=1}^{r} j^{2} a_{j} e^{-\sigma j}, \quad\left(\bar{B}_{n}^{(r)}\right)^{2}=\sum_{j=r}^{n} j^{2} a_{j} e^{-\sigma j}, \quad n=1,2, \ldots, \\
& \underline{\rho}_{n}^{(r)}=\sum_{j=1}^{r} j^{3} a_{j} e^{-\sigma j}, \quad \bar{\rho}_{n}^{(r)}=\sum_{j=r}^{n} j^{3} a_{j} e^{-\sigma j}, \quad n=1,2, \ldots
\end{aligned}
$$

Now we choose in (3.6) (resp. (3.7)) the parameter $\sigma$ equal to the unique solution of the equations (3.14) (resp. (3.15) below:

$$
\underline{M}_{n}^{(r)}(\sigma)=n
$$

and

$$
\bar{M}_{n}^{(r)}(\sigma)=n .
$$

The existence and uniqueness of the solution (in $\sigma$ ) of each of the two equations (3.14), (3.15), for any given $1 \leq r \leq n$ and $n=1,2, \ldots$, follow from the assumption that $a_{j}>0, j=1,2, \ldots$

The idea of the above choice of the free parameter $\sigma$, that goes back to Khintchine's book 29, is to evaluate the probabilities in (3.9), (3.10) when $n$ is the "most probable value" of the sums $\underline{Y}_{n}^{(r)}, \bar{Y}_{n}^{(r)}$. This makes the exponential factor in the expression of the normal density equal to 1 , which will enable us to obtain the principal term in the asymptotic expansions for the above probabilities. We will assume further on that $a \in \mathcal{F}(l), l>0$ and denote by $\underline{\sigma}_{n}^{(r)}, \bar{\sigma}_{n}^{(r)}$ the solutions of (3.14), 3.15) correspondingly.

Remark 1. In statistical physics, the idea of introducing a free parameter has its roots in the famous Darwin-Fowler asymptotic method developed in the 1920s for evaluating partition functions and mean values of occupation numbers. A good exposition of the method is given in [17, Ch. 6. In this method, the above quantities are expressed as complex integrals over a circle around the origin, of an arbitrary radius (= free parameter). Evaluating the integrals by the method of steepest descents, the free parameter is taken to be equal to the unique minimum point in $[0,1]$ of the integrand. In the preface to his book [29] Khintchine writes that the main novelty of his approach consists of replacing "the complicated analytical apparatus (the method of Darwin-Fowler) by...the well developed limit theorems of the theory of probability...that can form the analytical basis for all the computational formulas of statistical physics."

Finally, notice that a probabilistic method for the study of asymptotic problems arising in enumeration of permutations was quite independently suggested in the 1940 s by V. Goncharov. Subsequently, the method was extensively developed by generations of researchers who applied it to general combinatorial structures. The history of this line of research can be found in Kolchin's book [31].

Remark 2. As we already mentioned, a specific feature inherent in Khintchine's method is that the free parameter $\sigma$ depends on $n$, so that the random variables $X_{j}, j=1,2, \ldots$, form a triangular array. In the case of an array the conditions 
for a normal local limit theorem are not known. For this reason, starting from A. Khintchine (see [29], Ch. IV) and until the present time, the establishment of a local limit theorem for sums of random variables depending on a free parameter required sophisticated asymptotic analysis that differed from problem to problem. As examples, see (in chronological order) [19] of G. Freiman, [36, Ch. 2 of A. Postnikov, 21] of G. Freiman and J. Pitman, 33] of R. Mutafchiev, 31] of V. Kolchin, 20] of G. Freiman and B. Granovsky, and 22 of G. Freiman, A. Vershik and Yu. Yakubovitz. In particular, note that [12] of J. Deshouillers, G. Freiman and W. Moran gives an example of an array of random variables for which the local limit theorem fails though the Lyapunov condition holds.

Throughout the paper we will denote by $h, h_{i}, i=1,2, \ldots$, positive constants that appear in asymptotic formulae.

The following basic property of the solutions $\underline{\sigma}_{n}, \bar{\sigma}_{n}$ allows for the implementation of (2.8). The proof of it is similar to that of Lemma 3 in 20.

Lemma 1. Let $n \geq r \geq n^{\epsilon}$, for some $\epsilon>0$. Then

$$
\lim _{n \rightarrow \infty} \underline{\sigma}_{n}^{(r)}=0, \quad \lim _{n \rightarrow \infty} \bar{\sigma}_{n}^{(r)}=0
$$

It is clear that the straightforward application of the summation formula (2.8) to the sums in (3.11)-3.13) is not possible. Our subsequent asymptotic analysis extends the one in [20] in two different directions: from $c_{n}$ to $\underline{c}_{n}^{(r)}, \bar{c}_{n}^{(r)}, r=n^{\beta}, 0 \leq$ $\beta \leq 1$, and from the smooth case $a_{n} \sim n^{l-1}, l>0$ to the case $a_{n} \sim n^{l-1} L(n), l>0$. Our main tools will be the Euler integral test and a summation theorem of Abelian type.

Consider the function $f(x, \sigma)=x^{l} L(x) e^{-\sigma x}, x>0, \sigma \in R, l>0$.

If $\sigma>0$, then for sufficiently large $x>0$ and sufficiently small $\sigma$ the function $f$ has a maxima at the point $x=x(\sigma)$ which is the solution of the equation

$$
x L^{\prime}(x)+(l-\sigma x) L(x)=0
$$

Since (see [37], p. 7)

$$
\lim _{x \rightarrow \infty} \frac{x L^{\prime}(x)}{L(x)}=0
$$

for any s.v. function $L$, the asymptotic solution of (3.17) is given by $x \sim l \sigma^{-1}$, as $\sigma \rightarrow 0^{+}$. In the case $\sigma \leq 0$, the function $f$ is increasing in $x$ for sufficiently large $x>0$. Since we are interested in $r=n^{\beta}, 0<\beta<1$, Lemma 1 is valid. So, applying in both cases of $\sigma$ the integral test to the sums $\underline{M}_{n}(r), \bar{M}_{n}(r)$, we can rewrite (3.14) and (3.15) as

$$
n=\underline{M}_{n}(r) \sim \int_{1}^{r} f\left(x, \underline{\sigma}_{n}^{(r)}\right) d x
$$

$$
=\left(\left|\underline{\sigma}_{n}^{(r)}\right|\right)^{-(l+1)} \int_{\left|\underline{\sigma}_{n}^{(r)}\right|}^{r\left|\underline{\sigma}_{n}^{(r)}\right|} t^{l} L\left(\frac{t}{\left|\underline{\sigma}_{n}^{(r)}\right|}\right) \exp \left(-t \operatorname{sign}\left(\underline{\sigma}_{n}^{(r)}\right)\right) d t, \quad l>0, \quad n \rightarrow \infty
$$


and

$$
=\left(\left|\bar{\sigma}_{n}^{(r)}\right|\right)^{-(l+1)} \int_{r\left|\bar{\sigma}_{n}^{(r)}\right|}^{n\left|\bar{\sigma}_{n}^{(r)}\right|} t^{l} L\left(\frac{t}{\left|\bar{\sigma}_{n}^{(r)}\right|}\right) \exp \left(-\operatorname{tsgn}\left(\bar{\sigma}_{n}^{(r)}\right) d t, \quad l>0, \quad n \rightarrow \infty,\right.
$$

correspondingly. Next, in (2.8) we set $z=e^{-|\sigma|}$, so that $1-z \sim|\sigma|$, as $\sigma \rightarrow 0$, and apply the integral test to the sum $S(z)$ in the LHS, to obtain

$$
\int_{|\sigma|}^{\infty} t^{l} L\left(\frac{t}{|\sigma|}\right) e^{-t} d t \sim \Gamma(l+1) L\left(\frac{1}{|\sigma|}\right), \quad l>0, \quad \sigma \rightarrow 0 .
$$

Now we are in a position to establish asymptotic formulae for the three key parameters $\sigma, B^{2}$ and $\rho$ of the problem considered. To facilitate the understanding of the forthcoming asymptotic formulae we make the following:

Remark 3. Combining (3.19) and (3.20) with (3.21), it is easy to see that, for all $\beta, \quad 0<\beta<1$, both $\underline{\sigma}_{n}^{(r)}, \bar{\sigma}_{n}^{(r)}$ are

$$
\leq \tilde{L}(n) n^{-\frac{1}{l+1}}, \quad l>0,
$$

as $n \rightarrow \infty$, where $\tilde{L}$ is a s.v. function induced by the given s.v. function $L$. We will show in due course that $\frac{1}{l+1}$ is a threshold value in the context of the problem considered.

It is plain that our objective requires the derivation of asymptotic formulae for the integrals in the RHS's of (3.19), (3.20). The fact that $\sigma$ depends on $n$ does not allow the straightforward application of (2.8). To achieve the above goal we make use of the following fundamental fact in the theory of s.v. functions.

Proposition 1 ([10], Theorem 1.5.2, p. 22). For any $b>0$ and any s.v. L, the convergence

$$
\Phi(x, \lambda):=\frac{(x \lambda)^{\delta} L(\lambda x)}{x^{\delta} L(x)}-\lambda^{\delta} \rightarrow 0, \quad \text { as } \quad x \rightarrow \infty,
$$

is uniform in $\lambda \in[b, \infty)$, if $\delta<0$, and is uniform in $\lambda \in(0, b]$, if $\delta>0$ and if the function $x^{-\delta} L(x)$ is locally bounded on $[0, \infty)$.

Based on this result we now prove the following Abelian summation theorem which is a version of Proposition 4.1.2, p. 199 in [10].

Proposition 2. Let $0<b \leq \infty$ and let $b_{n} \rightarrow b, b_{n} z_{n} \rightarrow \infty, n \rightarrow \infty$. Then

$$
\int_{b_{n}}^{\infty} e^{-t} t^{l} L\left(t z_{n}\right) d t \sim L\left(b_{n} z_{n}\right) \int_{b_{n}}^{\infty} e^{-t} t^{l} d t, \quad l>0, \quad n \rightarrow \infty
$$

and, assuming the function $x^{-\delta} L(x)$ is locally bounded on $[0, \infty)$ for some $\delta>0$,

$$
\int_{0}^{b_{n}} e^{t} t^{l} L\left(t z_{n}\right) d t \sim L\left(b_{n} z_{n}\right) \int_{0}^{b_{n}} e^{t} t^{l} d t, \quad l>0, \quad n \rightarrow \infty .
$$

Proof. In Proposition 1 we set $x=z_{n} b_{n}, \lambda=t\left(b_{n}\right)^{-1}$ and write the identity

$$
L(\lambda x)=\Phi(x, \lambda) \lambda^{-\delta} L(x)+L(x)
$$

where $\Phi(x, \lambda)$ is as defined in (3.23). 
Since $\lambda \geq 1$ for all $t \geq b_{n}$, Proposition 1 gives

$$
\left|\int_{b_{n}}^{\infty} e^{-t} t^{l} \Phi\left(z_{n} b_{n}, \frac{t}{b_{n}}\right)\left(\frac{t}{b_{n}}\right)^{-\delta} d t\right| \leq \epsilon b_{n}^{\delta} \int_{b_{n}}^{\infty} e^{-t} t^{l-\delta} d t, \quad l>0,
$$

for all $\epsilon>0, \delta<0$ and all sufficiently large $n$. In (3.27) we have

$$
b_{n}^{\delta} \int_{b_{n}}^{\infty} e^{-t} t^{l-\delta} d t \sim \begin{cases}h, & \text { if } b<\infty, \\ e^{-b_{n}} b_{n}^{l+\delta}, & \text { if } b=\infty,\end{cases}
$$

where $h=b^{\delta} \int_{b}^{\infty} e^{-t} t^{l-\delta} d t<\infty$. Hence, the RHS of (3.27) tends to 0 , as $n \rightarrow \infty$. Now we substitute (3.26) into the LHS of (3.24) to get the first assertion. The assertion (3.25) is proved in the same manner, by applying Proposition 1 in the case $\delta>0$.

From now on, we set $\underline{r}=n \underline{\beta}, \bar{r}=n^{\bar{\beta}}$ and assume that the limit $d:=\lim _{n \rightarrow \infty} L(n)$, $0 \leq d \leq \infty$ exists. The forthcoming assertions tell us that the latter assumption plays a role only for the description of the behavior of the model at the critical point. In the case when the limit does not exist, the above description can be obtained in terms of partial limits of $L(n)$, as $n \rightarrow \infty$.

Proposition 2 will be repeatedly used for derivation of asymptotic formulae for the key parameters.

Lemma 2. (a) Let $(l+1)^{-1}<\underline{\beta} \leq 1$ and $0 \leq \bar{\beta}<(l+1)^{-1}$. Then

$$
\left.\underline{\sigma}_{n}^{(\underline{r})} \sim \bar{\sigma}_{n}^{(\bar{r})}\right) \sim(\Gamma(l+1))^{\frac{1}{l+1}} n^{-\frac{1}{l+1}} L_{1}(n), \quad l>0, \quad n \rightarrow \infty,
$$

where $L_{1}$ is a s.v. function determined by the s.v. function $L$ via the relationship

$$
\frac{1}{L_{1}\left(n^{l+1}\right)} \sim\left(L^{\frac{1}{l+1}}(n)\right)^{*}, \quad n \rightarrow \infty .
$$

(b) Let the function $x^{-\delta} L(x), \delta>0$ be locally bounded on $[0, \infty)$ and let $0<$ $\underline{\beta}<(l+1)^{-1}$ and $(l+1)^{-1}<\bar{\beta}<1$. Then

$$
\underline{\sigma}_{n}^{(\underline{r})} \sim-\frac{\underline{\gamma} \log n}{n \underline{\beta}}\left(1+\underline{\delta}_{n}\right), \quad l>0, \quad n \rightarrow \infty,
$$

where

$$
\underline{\gamma}=\underline{\gamma}_{n}=1-(l+1) \underline{\beta}-\frac{\log L(\underline{r})}{\log n}, \quad \underline{\delta}_{n}=\frac{\log (\underline{\gamma} \log n)}{\underline{\gamma} \log n}
$$

while

$$
\bar{\sigma}_{n}^{(\bar{r})} \sim \frac{\bar{\gamma} \log n}{n^{\bar{\beta}}}\left(1-\overline{\delta_{n}}\right), \quad l>0, \quad n \rightarrow \infty
$$

where

$$
\bar{\gamma}=\bar{\gamma}_{n}=(l+1) \bar{\beta}-1+\frac{\log L(\bar{r})}{\log n}, \quad \bar{\delta}_{n}=\frac{\log (\bar{\gamma} \log n)}{\bar{\gamma} \log n} .
$$

(c) Let $\beta=\bar{\beta}=(l+1)^{-1}$. Then the following three cases should be distinguished:

(i) If $0 \overline{<} d<\infty$, then

$$
\underline{\sigma}_{n}^{(\underline{r})} \sim \underline{A} n^{-\frac{1}{l+1}} L_{1}(n), \quad l>0, \quad n \rightarrow \infty,
$$

and

$$
\left.\bar{\sigma}_{n}^{(\bar{r})}\right) \sim \bar{A} n^{-\frac{1}{l+1}} L_{1}(n), \quad l>0, \quad n \rightarrow \infty,
$$


where $L_{1}$ is a s.v. function given by (3.30), while $\underline{A}, \bar{A}>0$ are the unique solutions of the equations

$$
\underline{A}^{l+1}=\int_{0}^{\underline{A} d^{\frac{1}{l+1}}} t^{l} e^{-t} d t
$$

and

$$
\bar{A}^{l+1}=\int_{\bar{A} d^{\frac{1}{l+1}}}^{\infty} t^{l} e^{-t} d t
$$

correspondingly.

(ii) If $d=0$, then $\bar{\sigma}_{n}^{(\bar{r})}$ is given by (3.29), while $\underline{\sigma}_{n}^{(\underline{r})}$ is given by (3.31), (3.32).

(iii) If $d=\infty$, then $\bar{\sigma}_{n}^{(\bar{r})}$ is given by (3.33), (3.34), while $\underline{\sigma}_{n}^{(\underline{r})}$ is given by (3.29), (3.30).

Proof. Since the equations (3.14), (3.15) have unique solutions (in $\sigma$ ), it suffices to check that the stated asymptotic formulae satisfy (3.19), (3.20).

Corollary 1. Let $\underline{\sigma}_{n}^{(\underline{r})}, \bar{\sigma}_{n}^{(\bar{r})}$ be given as in Lemma 2. Then, as $n \rightarrow \infty$,

$$
\begin{gathered}
\underline{B}^{2} \sim h \begin{cases}n\left(\underline{\sigma}_{n}^{(\underline{r})}\right)^{-1}, & \text { if }(l+1)^{-1}<\underline{\beta} \leq 1 \quad \text { or } \underline{\beta}=(l+1)^{-1}, d \neq 0, \\
n \underline{r}, \quad & \text { if } 0<\underline{\beta}<(l+1)^{-1} \quad \text { or } \underline{\beta}=(l+1)^{-1}, d=0,\end{cases} \\
\bar{B}^{2} \sim h \begin{cases}n\left(\bar{\sigma}_{n}^{(\bar{r})}\right)^{-1}, & \text { if } 0 \leq \bar{\beta}<(l+1)^{-1} \quad \text { or } \bar{\beta}=(l+1)^{-1}, d \neq \infty, \\
n \bar{r}, \quad & \text { if } \quad(l+1)^{-1}<\bar{\beta}<1 \quad \text { or } \bar{\beta}=(l+1)^{-1}, d=\infty,\end{cases} \\
\underline{\rho} \sim h \frac{\left(\underline{B}^{2}\right)^{2}}{n}, \quad \bar{\rho} \sim h \frac{\left(\bar{B}^{2}\right)^{2}}{n}, \quad n \rightarrow \infty .
\end{gathered}
$$

Remark 4. Lemma 2 and Corollary 1 show that $\beta=\frac{1}{l+1}$ is the critical value for the three key parameters $\sigma, B^{2}$ and $\rho$. We will see later on that this fact has a crucial influence on the asymptotic behavior of $\underline{c}_{n}^{(r)}$ and $\bar{c}_{n}^{(r)}$.

Corollary 1 implies that the following weaker form $\left(\rho=\sum_{k=1}^{n}\left(X_{k}-E X_{k}\right)^{3}<\right.$ $\sum_{k=1}^{n}\left|X_{k}-E X_{k}\right|^{3}$ ) of Lyapunov's condition (see [18], p. 278) holds for the sums $\underline{Y}_{n}^{(\underline{r})}$ and $\bar{Y}_{n}^{(\bar{r})}$ of random variables defined by $(\underline{3.8)}$ :

$$
\frac{\underline{\rho}}{\underline{B}^{3}} \rightarrow 0, \quad \frac{\bar{\rho}}{\bar{B}^{3}} \rightarrow 0, \quad n \rightarrow \infty .
$$

Recall that Lyapunov's condition is sufficient for the convergence to the normal law in the central limit theorem for independent random variables. Our next result shows that, for the triangular array considered, even a weaker form (3.42) of this condition is sufficient for the same convergence in the local limit theorem.

Theorem 2 (Local limit theorem). Let $a \in \mathcal{F}(l), l>0$, and let $\underline{\sigma}_{n}^{(\underline{r})}, \bar{\sigma}_{n}^{(\bar{r})}$ be as in Lemma 2. Then

$$
\begin{array}{ll}
\operatorname{Pr}\left(\underline{Y}_{n}^{(\underline{r})}=n\right) \sim\left(2 \pi \underline{B}^{2}\right)^{-\frac{1}{2}}, & n \rightarrow \infty, \\
\operatorname{Pr}\left(\bar{Y}_{n}^{(\bar{r})}=n\right) \sim\left(2 \pi \bar{B}^{2}\right)^{-\frac{1}{2}}, \quad n \rightarrow \infty .
\end{array}
$$


Proof. Our objective will be to derive the asymptotic behavior of the integrals $\underline{T}$ and $\bar{T}$ given by (3.9) and (3.10) respectively. The integrands in (3.9) and (3.10) are periodic with period 1 . So, for any $\alpha_{0}, 0<\alpha_{0} \leq 1 / 2$, the integrals can be written as

$$
\underline{T}=\underline{T}_{1}+\underline{T}_{2}, \quad \bar{T}=\bar{T}_{1}+\bar{T}_{2},
$$

where $\underline{T}_{1}=\underline{T}_{1}\left(\alpha_{0}\right), \bar{T}_{1}=\bar{T}_{1}\left(\alpha_{0}\right)$ and $T_{2}=T_{2}\left(\alpha_{0}\right), \bar{T}_{2}=\bar{T}_{2}\left(\alpha_{0}\right)$ are integrals of the integrands in (3.9), (3.10) over the sets $\left[-\alpha_{0}, \alpha_{0}\right]$ and $\left[-1 / 2,-\alpha_{0}\right] \cup\left[\alpha_{0}, 1 / 2\right]$ respectively. Following the approach of [21], 20], we will first show that for an appropriate choice of $\alpha_{0}=\alpha_{0}(n)$, the main contributions, as $n \rightarrow \infty$, to $\underline{T}$ and $\bar{T}$ come from $\underline{T}_{1}$, and $\bar{T}_{1}$ respectively. From (3.3)-(3.5) we have for $\alpha \in R$,

$$
\varphi_{j}(\alpha)=\sum_{k=0}^{\infty} \frac{\left(a_{j} e^{-j \sigma} e^{2 \pi i \alpha j}\right)^{k}}{k ! \exp \left(a_{j} e^{-j \sigma}\right)}=\exp \left(a_{j} e^{-j \sigma}\left(e^{2 \pi i \alpha j}-1\right)\right)
$$

and

$$
\begin{aligned}
& \underline{\varphi}^{(\underline{r})}(\alpha)=\exp \left(\sum_{j=1}^{\underline{r}} a_{j} e^{-j \underline{\sigma}_{n}^{(\underline{r})}}\left(e^{2 \pi i \alpha j}-1\right)\right), \\
& \bar{\varphi}^{(\bar{r})}(\alpha)=\exp \left(\sum_{j=\bar{r}}^{n} a_{j} e^{-j \bar{\sigma}_{n}^{(\bar{r})}}\left(e^{2 \pi i \alpha j}-1\right)\right) .
\end{aligned}
$$

Substituting the Taylor expansion (in $\alpha$ )

$$
e^{2 \pi i \alpha j}-1=2 \pi i \alpha j-2 \pi^{2} \alpha^{2} j^{2}+O\left(\alpha^{3} j^{3}\right), \quad \text { as } \alpha \rightarrow 0,
$$

in (3.47), (3.48) and employing (3.14), (3.15), gives

$$
\begin{aligned}
& \underline{\varphi}^{(\underline{r})}(\alpha) e^{-2 \pi i \alpha n}=\exp \left(-2 \pi^{2} \alpha^{2} \underline{B}^{2}+O\left(\alpha^{3} \underline{\rho}\right)\right), \quad \text { as } \alpha \rightarrow 0, \\
& \bar{\varphi}^{(\bar{r})}(\alpha) e^{-2 \pi i \alpha n}=\exp \left(-2 \pi^{2} \alpha^{2} \bar{B}^{2}+O\left(\alpha^{3} \bar{\rho}\right)\right), \quad \text { as } \alpha \rightarrow 0 .
\end{aligned}
$$

We write now

$$
\underline{\alpha}_{0}^{3} \underline{\rho}=\left(\underline{\alpha}_{0} \underline{B}\right)^{3} \frac{\underline{\rho}}{\underline{B}^{3}}, \quad \bar{\alpha}_{0}^{3} \bar{\rho}=\left(\bar{\alpha}_{0} \bar{B}\right)^{3} \frac{\bar{\rho}}{\bar{B}^{3}}
$$

to conclude that, by (3.42), there exist $\underline{\alpha}_{0}=\underline{\alpha}_{0}(n), \bar{\alpha}_{0}=\bar{\alpha}_{0}(n)$ such that

$$
\lim _{n \rightarrow \infty} \underline{\alpha}_{0} \underline{B}=\lim _{n \rightarrow \infty} \bar{\alpha}_{0} \bar{B}=+\infty
$$

and

$$
\lim _{n \rightarrow \infty} \underline{\alpha}_{0}^{3} \underline{\rho}=\lim _{n \rightarrow \infty} \bar{\alpha}_{0}^{3} \bar{\rho}=0 .
$$

We see from (3.54) that $\underline{\alpha}_{0}, \bar{\alpha}_{0} \rightarrow 0, n \rightarrow \infty$, because $\underline{\rho}, \bar{\rho} \rightarrow \infty, n \rightarrow \infty$, by (3.41). Also note the fact that (3.49) holds for all $\alpha \in\left[-\underline{\alpha}_{0}, \underline{\alpha}_{0}\right] \cup\left[-\bar{\alpha}_{0}, \bar{\alpha}_{0}\right]$. As a 
result, we arrive at the asymptotic formulae for the integrals $\underline{T}_{1}, \bar{T}_{1}$ :

$$
\begin{aligned}
\underline{T}_{1} & \sim \int_{\underline{-}_{0}}^{\underline{\alpha}_{0}} \exp \left(-2 \pi^{2} \alpha^{2} \underline{B}^{2}\right) d \alpha \\
& =\frac{1}{2 \pi \underline{B}} \int_{-2 \pi \alpha_{0} \underline{B}}^{2 \pi \alpha_{0} \underline{B}} \exp \left(-\frac{z^{2}}{2}\right) d z \sim \frac{1}{\sqrt{2 \pi \underline{B}^{2}}}, \quad n \rightarrow \infty, \\
\bar{T}_{1} & \sim \int_{-\bar{\alpha}_{0}}^{\bar{\alpha}_{0}} \exp \left(-2 \pi^{2} \alpha^{2} \bar{B}^{2}\right) d \alpha \\
& =\frac{1}{2 \pi \bar{B}} \int_{-2 \pi \bar{\alpha}_{0} \bar{B}}^{2 \pi \bar{\alpha}_{0} \bar{B}} \exp \left(-\frac{z^{2}}{2}\right) d z \sim \frac{1}{\sqrt{2 \pi \bar{B}^{2}}}, \quad n \rightarrow \infty .
\end{aligned}
$$

Now we turn to the estimation, as $n \rightarrow \infty$, of the integrals $\underline{T}_{2}, \bar{T}_{2}$. We have

$$
\left|\underline{T}_{2}\right|=2\left|\int_{\underline{\alpha}_{0}}^{1 / 2} \underline{\varphi}^{(\underline{r})}(\alpha) e^{-2 \pi i \alpha n} d \alpha\right|, \quad\left|\bar{T}_{2}\right|=2\left|\int_{\bar{\alpha}_{0}}^{1 / 2} \bar{\varphi}^{(\bar{r})}(\alpha) e^{-2 \pi i \alpha n} d \alpha\right| .
$$

It follows from (3.47), (3.48) that

$$
\begin{aligned}
& \left|\underline{\varphi}^{(\underline{r})}(\alpha)\right|=\exp \left(-2 \sum_{j=1}^{\underline{r}} a_{j} e^{-j \underline{\sigma}_{n}^{(\underline{r})}} \sin ^{2} \pi \alpha j\right), \quad \alpha \in R, \\
& \left|\bar{\varphi}^{(\bar{r})}(\alpha)\right|=\exp \left(-2 \sum_{j=\bar{r}}^{n} a_{j} e^{-j \overline{\left.\sigma_{n}^{(}\right)}} \sin ^{2} \pi \alpha j\right), \quad \alpha \in R .
\end{aligned}
$$

We denote

$$
\begin{array}{ll}
\underline{V}_{n}^{(\underline{r})}(\alpha)=2 \sum_{j=1}^{\underline{r}} a_{j} e^{-j \underline{\sigma}_{n}^{(\underline{r})}} \sin ^{2} \pi \alpha j, & \underline{\alpha}_{0} \leq \alpha \leq 1 / 2, \\
\bar{V}_{n}^{(\bar{r})}(\alpha)=2 \sum_{j=\bar{r}}^{n} a_{j} e^{-j \bar{\sigma}_{n}^{(\bar{r})}} \sin ^{2} \pi \alpha j, & \bar{\alpha}_{0} \leq \alpha \leq 1 / 2 .
\end{array}
$$

For the sake of estimating the sums $\underline{V}_{n}^{(\underline{r})}, \bar{V}_{n}^{(\bar{r})}$ we make use of the following inequality proven in [21]:

$$
2 \sum_{j=p}^{p+k-1} \sin ^{2} \pi \alpha j \geq \frac{k}{2} \min \left\{1,(\alpha k)^{2}\right\}, \quad|\alpha| \leq 1 / 2, \quad \forall k \geq 2, p \geq 1 .
$$

We set

$$
\underline{\alpha}_{0}^{2}=\frac{\log ^{4}\left(\underline{B}^{2}\right)}{\underline{B}^{2}}, \quad \bar{\alpha}_{0}^{2}=\frac{\log ^{4}\left(\bar{B}^{2}\right)}{\bar{B}^{2}}
$$

and apply (3.62) with

$$
\underline{k}=l\left(\left|\underline{\sigma}_{n}^{(\underline{r})}\right|\right)^{-1}, \quad \bar{k}=l\left(\left|\bar{\sigma}_{n}^{(\bar{r})}\right|\right)^{-1}
$$

and different $\underline{p}, \bar{p}$. (Note that under the choice (3.63) of $\underline{\alpha}_{0}, \bar{\alpha}_{0}$, the conditions (3.53), (3.54) indeed hold.) Treating separately the cases (a), (b) and (c) in Lemma 2, we are able to show that

$$
e^{-\underline{V}_{n}^{(\underline{r})}(\alpha)}=o\left(\underline{B}^{-1}\right), \quad \underline{\alpha}_{0} \leq \alpha \leq 1 / 2, \quad n \rightarrow \infty,
$$


and

$$
e^{-\bar{V}_{n}^{(\bar{r})}(\alpha)}=o\left(\bar{B}^{-1}\right), \quad \overline{\alpha_{0}} \leq \alpha \leq 1 / 2, \quad n \rightarrow \infty
$$

Corollary 2 (Asymptotic formulae for $\left.\underline{c}_{n}^{(\underline{r})}, \bar{c}_{n}^{(\bar{r})}\right)$. Let $\underline{r}=n \underline{\beta}, 0<\underline{\beta} \leq 1$, and $\bar{r}=n^{\bar{\beta}}, 0 \leq \bar{\beta}<1$. Then

$$
\begin{array}{ll}
\underline{c}_{n}^{(\underline{r})} \sim\left(2 \pi \underline{B}^{2}\right)^{-\frac{1}{2}} \exp \left(\underline{S}_{n}^{(\underline{r})}\left(e^{-\underline{\sigma}_{n}^{(\underline{r})}}\right)+n \underline{\sigma}_{n}^{(\underline{r})}\right), & n \rightarrow \infty, \\
\bar{c}_{n}^{(\bar{r})} \sim\left(2 \pi \bar{B}^{2}\right)^{-\frac{1}{2}} \exp \left(\bar{S}_{n}^{(\bar{r})}\left(e^{-\bar{\sigma}_{n}^{(\bar{r})}}\right)+n \bar{\sigma}_{n}^{(\bar{r})}\right), \quad n \rightarrow \infty,
\end{array}
$$

where

$$
\begin{gathered}
\underline{S}_{n}^{(\underline{r})}\left(e^{-\underline{\sigma}_{n}^{(\underline{r})}}\right) \sim h \frac{n^{2}}{\underline{B}^{2}}, \\
\bar{S}_{n}^{(\bar{r})}\left(e^{-\bar{\sigma}_{n}^{(\bar{r})}}\right) \sim h \frac{n^{2}}{\bar{B}^{2}}, \quad n \rightarrow \infty .
\end{gathered}
$$

Proof. By Theorem 1 and (3.6), (3.7) we get the asymptotic expressions (3.66), (3.67), while (3.68) is obtained with the help of the integral test, Proposition 2, Lemma 2 and Corollary 1.

Theorem 3 (The limiting behavior of $\left.\underline{d}_{n}^{(\underline{r})}, \bar{d}_{n}^{(\bar{r})}\right)$. Denote $\underline{\underline{c}}_{n}^{(n)}=c_{n}$.

(i) Let $\underline{r}=n \underline{\beta}, \quad 0 \leq \underline{\beta} \leq 1$. Then

$$
\lim _{n \rightarrow \infty} \underline{d}_{n}^{(\underline{r})}= \begin{cases}0, & \text { if } \quad 0 \leq \underline{\beta}<(l+1)^{-1} \quad \text { or } \underline{\beta}=(l+1)^{-1}, d<\infty, \\ 1, & \text { if } \quad(l+1)^{-1}<\underline{\beta} \leq 1 \quad \text { or } \underline{\beta}=(l+1)^{-1}, d=\infty .\end{cases}
$$

(ii) Let $\bar{r} \geq 2$. Then

$$
\lim _{n \rightarrow \infty} \bar{d}_{n}^{(\bar{r})}= \begin{cases}0, & \text { if } \quad \bar{r}=n^{\bar{\beta}}, \quad 0<\bar{\beta} \leq 1, \\ \exp \left(-\sum_{j=1}^{\bar{r}-1} a_{j}\right), & \text { if } \bar{r} \geq 2 \quad \text { is a fixed number. }\end{cases}
$$

Proof. (i) Denote

$$
\Delta_{n}^{(\underline{r})}=\underline{S}_{n}^{(\underline{r})}\left(e^{-\underline{\sigma}_{n}^{(\underline{r})}}\right)-\underline{S}_{n}^{(n)}\left(e^{-\underline{\sigma}_{n}^{(n)}}\right)+n\left(\underline{\sigma}_{n}^{(\underline{r})}-\underline{\sigma}_{n}^{(n)}\right) .
$$

Our objective will be to demonstrate that

$$
\lim _{n \rightarrow \infty} \Delta_{n}^{(\underline{r})}=\left\{\begin{array}{lll}
-\infty, & \text { if } \quad 0<\underline{\beta}<(l+1)^{-1} & \text { or } \quad \underline{\beta}=(l+1)^{-1}, d<\infty \\
0, & \text { if } \quad(l+1)^{-1}<\underline{\beta} \leq 1 & \text { or } \underline{\beta}=(l+1)^{-1}, d=\infty .
\end{array}\right.
$$

The first part of (3.72) follows in a straightforward way from the preceding asymptotic analysis. In the case corresponding to the second part,

$$
\underline{\sigma}_{n}^{(n)} \sim \underline{\sigma}_{n}^{(\underline{r})}, \quad n \rightarrow \infty,
$$

and, consequently,

$$
\underline{S}_{n}^{(\underline{r})}\left(e^{-\underline{\sigma}_{n}^{(r)}}\right) \sim \underline{S}_{n}^{(n)}\left(e^{-\underline{\sigma}_{n}^{(n)}}\right), \quad n \rightarrow \infty .
$$

Therefore, here a more subtle analysis is required. From the identity

$$
\underline{M}_{n}^{(\underline{r})}\left(\underline{\sigma}_{n}^{(\underline{r})}\right)-\underline{M}_{n}^{(n)}\left(\underline{\sigma}_{n}^{(n)}\right)=0, \quad \underline{r}=n^{\beta}, 1 \geq \beta \geq \frac{1}{l+1}, \quad n=2,3, \ldots,
$$


and the fact that $\underline{\sigma}_{n}^{(n)}>\underline{\sigma}_{n}^{(\underline{r})} \geq 0$, we derive that $n\left(\underline{\sigma}_{n}^{(n)}-\underline{\sigma}_{n}^{(\underline{r})}\right) \rightarrow 0, n \rightarrow \infty$. Similarly, it can be proven that in the case considered

$$
\underline{S}_{n}^{(\underline{r})}\left(e^{-\underline{\sigma}_{n}^{(\underline{r})}}\right)-\underline{S}_{n}^{(n)}\left(e^{-\underline{\sigma}_{n}^{(n)}}\right) \rightarrow 0, n \rightarrow \infty .
$$

Combining this with the asymptotic formulae in Corollary 1 and Corollary 2, proves the second part of (3.69).

(ii) We outline only the proof of the second part of (3.70). We have for a fixed $\bar{r} \geq 2$,

$$
\bar{\sigma}_{n}^{(\bar{r})} \sim \bar{\sigma}_{n}^{(1)}, \quad n \rightarrow \infty, \quad \text { and } \quad \lim _{n \rightarrow \infty} n\left(\bar{\sigma}_{n}^{(\bar{r})}-\bar{\sigma}_{n}^{(1)}\right)=0
$$

Next we write

$$
\begin{aligned}
\bar{S}_{n}^{(\bar{r})} & \left(e^{-\bar{\sigma}_{n}^{(\bar{r})}}\right)-\underline{S}_{n}^{(n)}\left(e^{-\bar{\sigma}_{n}^{(1)}}\right) \\
& =\sum_{j=1}^{n} a_{j} e^{-\bar{\sigma}_{n}^{(1)} j}\left(e^{-\left(\bar{\sigma}_{n}^{(\bar{r})}-\bar{\sigma}_{n}^{(1)}\right) j}-1\right)-\sum_{j=1}^{\bar{r}-1} a_{j} e^{-\bar{\sigma}_{n}^{(\bar{r})} j} .
\end{aligned}
$$

Since $\bar{\sigma}_{n}^{(1)}>\bar{\sigma}_{n}^{(\bar{r})} \geq 0$ and

$$
e^{-\left(\bar{\sigma}_{n}^{(\bar{r})}-\bar{\sigma}_{n}^{(1)}\right) j}-1=\left(\bar{\sigma}_{n}^{(1)}-\bar{\sigma}_{n}^{(\bar{r})}\right) j\left(1-\delta_{n}\right), \quad 1 \leq j \leq n,
$$

where $\delta_{n}=\delta_{n}(j) \rightarrow 0, \quad n \rightarrow \infty$, uniformly in $1 \leq j \leq n$, we get the desired claim.

\section{Application 1: Reversible coagulation-fragmentation processes}

We follow the formulation of the model given in [13. A population of $n$ particles is partitioned into groups of various sizes that undergo stochastic evolutions (in time) of coagulation and fragmentation. There are only two possible interactions: coagulation of two groups into one, and fragmentation of one group into two groups. The process of coagulation-fragmentation (CFP) is a time-homogeneous interacting particle system $\varphi_{t}, t \geq 0$, defined as follows. For a given $n$, denote by $\eta=\left(k_{1}, \ldots, k_{n}\right)$ a partition of the whole population $n$ into $k_{i}$ groups of size $i, i=1,2, \ldots, n$, where the numbers of groups $k_{i} \geq 0$ are subject to the condition:

$$
\sum_{i=1}^{n} i k_{i}=n
$$

called the total mass conservation law. The finite set $\Omega_{n}=\{\eta\}$ of all partitions of $n$ is the state space of the process $\varphi_{t}, t \geq 0$. The rates of the infinitesimal (in time) transitions (= flips) are assumed to depend only on the sizes of the interacting groups, and are given by two functions $\psi$ and $\phi$ :

(1) For $i$ and $j$ such that $i+j \leq n$, the rate of coagulation, $(i, j) \rightarrow(i+j)$, of two groups of sizes $i$ and $j$ into one group of size $i+j$, equals $\psi(i, j)$.

(2) The rate of fragmentation, $(i+j) \rightarrow(i, j)$, of a group of size $i+j$ into two groups of sizes $i$ and $j$, equals $\phi(i, j)$.

Hereafter, we refer to the coagulation and fragmentation rates $\psi$ and $\phi$ as intensities. The intensities are required to satisfy $\psi(i, j)=\psi(j, i) \geq 0$ and $\phi(i, j)=\phi(j, i) \geq 0$. 
We also make the natural assumption that the total intensities of merging $\Psi(i, j ; \eta)$ and splitting $\Phi(i, j ; \eta)$ at a configuration $\eta \in \Omega_{n}$ are given by

$$
\begin{gathered}
\Psi(i, j ; \eta)=\Psi\left(i, j ; k_{i}, k_{j}\right)=\psi(i, j)\left(k_{i} k_{j}\right)^{\gamma}, \quad i \neq j, \quad 2 \leq i+j \leq n, \\
\Psi(i, i ; \eta)=\Psi\left(i, i ; k_{i}, k_{i}\right)=\psi(i, i)\left(k_{i}\left(k_{i}-1\right)\right)^{\gamma}, \quad 2 \leq 2 i \leq n, \\
\Phi(i, j ; \eta)=\Phi\left(i, j ; k_{i}, k_{j}\right)=\phi(i, j)\left(k_{i+j}\right)^{\gamma}, \quad 2 \leq i+j \leq n,
\end{gathered}
$$

where $\gamma>0$. Note that the case $\gamma=1$ corresponds to the mass action kinetics.

In this paper, we study only reversible CFP's with nonzero intensities. It is known ([13, [30]) that the class of such processes is characterized by the following property of the ratio of their intensities:

$$
\frac{\psi(i, j)}{\phi(i, j)}=\frac{a_{i+j}}{a_{i} a_{j}}, \quad 2 \leq i+j \leq n,
$$

where $a=\left\{a_{i}=a(i)\right\}, i=1,2, \ldots$, is a positive function. It is also known ([13], [30]) that, under (4.3), the unique invariant measure $\mu_{n}$ on $\Omega_{n}$ is given by

$$
\mu_{n}(\eta)=C_{n} \frac{a_{1}^{k_{1}} a_{2}^{k_{2}} \ldots a_{n}^{k_{n}}}{\left(k_{1} !\right)^{\gamma}\left(k_{2} !\right)^{\gamma}, \ldots\left(k_{n} !\right)^{\gamma}}, \quad \eta=\left(k_{1}, \ldots, k_{n}\right) \in \Omega_{n} .
$$

Here $C_{n}^{-1}(a)=C_{n}^{-1}:=c_{n}$ is the partition function for the probability measure $\mu_{n}, n \geq 1$ :

$$
c_{n}=\sum_{\eta \in \Omega_{n}} \frac{a_{1}^{k_{1}} a_{2}^{k_{2}} \ldots a_{n}^{k_{n}}}{\left(k_{1} ! k_{2} ! \ldots k_{n} !\right)^{\gamma}}, \quad \eta=\left(k_{1}, \ldots, k_{n}\right) \in \Omega_{n} .
$$

The measure $\mu_{n}$ is the steady state of the reversible CFP considered. So, (4.4) tells us that for a fixed $n$, the steady state is determined by $n$ values of the function $a$. In view of this, it is natural to call $a$ the parameter function of the process. Note that in contrast to the above, the transient behavior of the CFP's considered depends on the intensities $\psi$ and $\phi$, rather than on their ratios.

Remark 5. The measure $\mu_{n}$ is invariant under the following transformation of the parameter function $a$. Define the family of operators $H_{h}, h>0$ on a set of parameter functions $a$ :

$$
\left(H_{h} a\right)(j)=h^{j} a_{j}, \quad j=1,2, \ldots, \quad h>0 .
$$

It follows from (4.1) and (4.4) that (with the obvious abuse of notation)

$$
H_{h} \mu_{n}=\mu_{n}, \quad h>0 .
$$

This says that all results of the present paper are extended to the class of parameter functions $\left\{H_{h} a: h>0, a \in \mathcal{F}(l), l>0\right\}$. (4.6) also explains the possibility of introducing a free parameter for the treatment of problems related to $\mu_{n}$.

Our study is devoted exclusively to the steady state of the above CFP's, in the case when in (4.4), $\gamma=1$ and $n \rightarrow \infty$. Treating $S$ given by (2.7) as a generating function of the positive sequence $\left\{a_{n}\right\}_{1}^{\infty}$, such that the radius of convergence of the series (2.7) equals 1, it is known (see e.g. [13]) that the sequence $\left\{c_{n}\right\}_{0}^{\infty}$ in (4.5) is generated by the function $g$ defined by

$$
g(z)=e^{S(z)}=\sum_{n=0}^{\infty} c_{n} z^{n}, \quad|z|<1 .
$$


To formulate the problem of clustering in the setting of CFP, we define on the probability space $\left(\Omega_{n}, \mu_{n}\right)$ the random variables $K_{i}=K_{i}^{(n)}(\eta)=$ the number of groups of size $i, i=1, \ldots, n$, in a random partition $\eta \in \Omega_{n}$, and let $\bar{q}_{n}=\bar{q}_{n}(\eta)$, (resp. $\left.\underline{q}_{n}=\underline{q}_{n}(\eta)\right)$ be the size of the largest (resp. smallest) group. We will be interested in the probabilities $\operatorname{Pr}\left(\bar{q}_{n} \leq r\right)$ and $\operatorname{Pr}\left(\underline{q}_{n} \geq r\right)$. Making use of the notation in (2.13), we have

$$
\begin{aligned}
& \underline{c}_{n}^{(r)}=\sum_{\eta \in \Omega_{n}: \bar{q}_{n}(\eta) \leq r} \frac{a_{1}^{k_{1}} a_{2}^{k_{2}} \ldots a_{n}^{k_{n}}}{k_{1} ! k_{2} ! \ldots k_{n} !}, \\
& \bar{c}_{n}^{(r)}=\sum_{\eta \in \Omega_{n}: \underline{q}_{n}(\eta) \geq r} \frac{a_{1}^{k_{1}} a_{2}^{k_{2}} \ldots a_{n}^{k_{n}}}{k_{1} ! k_{2} ! \ldots k_{n} !} .
\end{aligned}
$$

This gives

$$
\operatorname{Pr}\left(\bar{q}_{n} \leq r\right)=\frac{\underline{c}_{n}^{(r)}}{c_{n}}, \quad \operatorname{Pr}\left(\underline{q}_{n} \geq r\right)=\frac{\bar{c}_{n}^{(r)}}{c_{n}} .
$$

We assume now that $a \in \mathcal{F}(l), l>0$, and $r=n^{\beta}, 0 \leq \beta \leq 1$. Then Theorem 3 admits the following interpretation:

$$
\lim _{n \rightarrow \infty} \operatorname{Pr}\left(\bar{q}_{n} \leq n^{\beta}\right)=\left\{\begin{array}{lll}
0, & \text { if } \quad 0 \leq \beta<(l+1)^{-1} & \text { or } \quad \beta=(l+1)^{-1}, d<\infty, \\
1, & \text { if } \quad(l+1)^{-1}<\beta \leq 1 \quad \text { or } \quad \beta=(l+1)^{-1}, d=\infty,
\end{array}\right.
$$

while

$$
\lim _{n \rightarrow \infty} \operatorname{Pr}\left(\underline{q}_{n} \geq r\right)= \begin{cases}0, & \text { if } r=n^{\beta}, \quad 0<\beta \leq 1, \\ \exp \left(-\sum_{j=1}^{r-1} a_{j}\right), & \text { if } \quad r \geq 2 \quad \text { is a fixed number. }\end{cases}
$$

Remark 6. (4.10) identifies $n^{\frac{1}{l+1}}$ as the threshold for the limiting distribution of the size of the largest cluster, in the sense that

$$
\frac{1}{l+1}=\inf \left\{\beta: \operatorname{Pr}\left(\bar{q}_{n} \leq n^{\beta}\right)=1\right\} .
$$

We discuss the phenomenon in more detail in Remark 8 , in the context of random combinatorial structures.

To reveal the picture of clustering at the equilibrium of the CFP's considered, we establish one more fact.

Theorem 4. Let $a \in \mathcal{F}(l), l>0$. Then

$$
\lim _{n \rightarrow \infty} \operatorname{Pr}\left(n^{\frac{1}{1+1}-\epsilon}<\bar{q}_{n}<n^{\frac{1}{1+1}+\epsilon}\right)=1, \quad \forall \epsilon>0 .
$$

(ii) For all $p$ such that $n^{\epsilon} \leq p \leq n^{\beta}$, with $\beta<\frac{1}{l+1}$ and $\epsilon>0$,

$$
\lim _{n \rightarrow \infty} \operatorname{Pr}\left(K_{p}=0\right)= \begin{cases}1, & \text { if } 0<l<1 \\ e^{-d}, & \text { if } l=1 \\ 0, & \text { if } l>1\end{cases}
$$


(iii) For any two $s$-tuples of integers $p_{1}, \ldots, p_{s} \geq 1$ and $k_{1}, \ldots, k_{s} \geq 0$,

$$
\lim _{n \rightarrow \infty} \operatorname{Pr}\left(K_{p_{1}}=k_{1}, \ldots, K_{p_{s}}=k_{s}\right)=\prod_{j=1}^{s} \frac{a_{p_{j}}^{k_{j}}}{k_{j} !} e^{-a_{p_{j}}} .
$$

Proof. (4.12) follows immediately from (4.10). Next, we have

$$
\operatorname{Pr}\left(K_{p}=0\right)=\sum_{\eta \in \Omega_{n}: k_{p}=0} \mu_{n}(\eta):=\frac{c_{n, p}}{c_{n}}, \quad 1 \leq p \leq n .
$$

Denote by $\sigma_{n, p}, B_{n, p}^{2}$, the key parameters of the asymptotics of $c_{n, p}$. Namely, $\sigma_{n, p}$ is the unique solution in $\sigma$ of the equation

$$
\underline{M}_{n}^{(n)}(\sigma)-p a_{p} e^{-\sigma p}=n, \quad l>0,
$$

and $B_{n, p}^{2}$ is defined correspondingly. Then in the case $1 \leq p<n^{\beta}, 0<\beta<\frac{1}{l+1}$, we have

$$
\begin{aligned}
& \sigma_{n, p} \sim \underline{\sigma}_{n}^{(n)} \sim(\Gamma(l+1))^{\frac{1}{l+1}} n^{-\frac{1}{l+1}} L_{1}(n), \\
& B_{n, p} \sim \underline{B}_{n}^{(n)}, \quad n \rightarrow \infty, \quad \sigma_{n, p}<\underline{\sigma}_{n}^{(n)} .
\end{aligned}
$$

By the reasoning used for the proof of the second part of (3.70), we get from (4.17), for $n \rightarrow \infty$,

$0 \leq n\left(\sigma_{n, p}-\underline{\sigma}_{n}^{(n)}\right) \rightarrow 0$, and $S_{n, p}\left(e^{-\sigma_{n, p}}\right)-\underline{S}_{n}^{(n)}\left(e^{-\underline{\sigma}_{n}^{(n)}}\right)+a_{p} e^{-p \sigma_{n, p}} \rightarrow 0, l>0$, which implies 4.13). In the same manner we obtain

$$
\lim _{n \rightarrow \infty} \operatorname{Pr}\left(K_{p}=k_{p}\right)=\frac{a_{p}^{k_{p}}}{k_{p} !} \operatorname{Pr}\left(K_{p}=0\right),
$$

which implies 4.14 for $s=1$. For general $s$ the proof is similar.

Remark 7. (4.14) tells us that the random variables depicting numbers of groups of fixed sizes become independent, as $n \rightarrow \infty$. This fact is in accordance with the general principle of asymptotic independence of particles in mean-field models, that is commonly accepted (but not rigorously proved) in statistical physics (see 13. and the references therein). In the case $a \in \mathcal{F}(l), l \leq 0$, the independence principle was broadly discussed in the context of random combinatorial structures (see Remark 8).

Now we are in a position to provide a verbal description of the striking feature of clustering for large $n$, in the case $a \in \mathcal{F}(l), l>0$.

- With probability 1 , there are no clusters(=groups) of sizes greater than $O\left(n^{\beta}\right), \beta>\frac{1}{l+1}$. Moreover, with probability 1 , the size of the largest group lies in the interval $\left[n^{\frac{1}{l+1}-\epsilon}, n^{\frac{1}{l+1}+\epsilon}\right], \epsilon>0$.

On the other hand:

- The $n$ particles are partitioned into groups of sizes not greater than $O\left(n^{\frac{1}{l+1}}\right)$ in such a way that

(i) with a positive probability there are groups of any fixed size;

(ii) the limiting probability of having a group of a size $p, p \in\left[n^{\epsilon}, n^{\beta}\right]$, $\epsilon>0,0<\beta<\frac{1}{l+1}$, equals $0,1-e^{-d}$ or 1 , if $0<l<1, l=1$ or $l>1$ respectively. 
Summing up the aforementioned picture, we conclude that for large $n$ the distribution of clusters induced by the measure $\mu_{n}$ has a threshold $n^{\frac{1}{l+1}}$.

Historical remarks. It is generally accepted that the mathematical chapter of the history of CFP's traces back to the 1917 paper [38] by M. Smoluchowski. In this seminal work the mathematical theory of the process of pure coagulation of molecules of colloids was proposed. A deep discussion of the physical context and implications of Smoluchowski model was presented in Ch. III of the classical work by S. Chandrasekhar (1943) reprinted in [40]. Observe that coagulation was treated by Smoluchowski as a deterministic process. In the framework of this approach, there was derived in [38 an infinite system of differential equations describing the evolution in time of the concentration of molecules of sizes $1,2, \ldots$. (Note that some authors mistakenly attribute the equations to another paper by Smoluchowski published in 1916.)

Subsequently, the equations, after being generalized to allow also fragmentations of particles, became famous as a general model for processes of grouping and splitting in numerous fields of applications. Efforts of generations of researchers were devoted to the intriguing mathematical problems of existence, uniqueness and asymptotic behavior (in time and in the number of particles) of the solutions.

It was understood a long time ago that a stochastic context could be attributed to Smoluchowski's equations (SE). Corresponding stochastic models were independently reintroduced, under different names, in different fields of applications (see for details the review [2]). The paper [3] attributes to A. Marcus the first stochastic model for pure coagulation, called the Marcus-Lushnikov process (MLP). Extensive study of MLP was concentrated around two subjects: the gelation phenomena and the relation of MLP to SE. ("Gelation" is the name for the phase transition exhibited by the formation of a giant cluster that causes the violation of the total mass conservation law (4.1)). The main approach to these problems is based on treating the MLP as the stochastic coalescent. A program for investigating the relationship between MLP (= stochastic coalescent) and SE was outlined by D. Aldous in [2]. Recent progress in this direction was made by J. Norris in [34, who proved that under certain conditions a sequence of stochastic coalescents converges weakly to the solution of the SE. The theory of coalescents as a tool to study limits of coagulation models as $n \rightarrow \infty$, was developed by J. Pitman et al. (see e.g. 115]).

Parallel to this line of research, Monte Carlo algorithms based on MLP were developed for the numerical treatment of SE (see [14]) and references therein).

P. Whittle [41] proposed a reversible Markov process as a model for Flory's theory of polymerization developed in the 1940s. As a result, a system of SE (in the presence of fragmentation) was rediscovered for both deterministic and stochastic contexts (see also [42]). M. Aizenmann and T. Bak 1], also motivated by Flory's theory, proved that for the continuous (in space) version of SE with constant kernels of coagulation and fragmentation, the free energy of the system decays exponentially as time $t \rightarrow \infty$. This important fact established the validity of Boltzmann's H-theorem for the time evolution of the system described by SE. Note that a general fact of increasing entropy for SE with kernels obeying the deterministic reversibility condition was independently proven in [41].

The explicit formulation of a CFP as a Markov process on the set of partitions appears in the monograph [30], Ch. 8, by F. Kelly, which contains also the expression (4.4) for the equilibrium distribution of reversible CFP's in the case $\gamma=1$. (In [30] 
the model is called a clustering process.) The above formulation was reintroduced by S. Gueron in [24] in the context of animal grouping. As far as we know, Gueron, [24], was the first to notice that SE are obtained from the Kolmogorov forward equations for the expected numbers of groups, by neglecting correlations among the numbers $K_{p}$ of groups of different sizes $p=1,2, \ldots$. R. Durrett, B. Granovsky and S. Gueron [13] studied the asymptotic behavior (in $n$ ) of $E K_{p}$ and $\operatorname{cov}\left(K_{p_{1}}, K_{p_{2}}\right)$ at the steady state (4.5) with $\gamma=1$ and an arbitrary parameter function $a$. They showed that

$$
\lim _{n \rightarrow \infty} \operatorname{cov}\left(K_{p_{1}}, K_{p_{2}}\right)=0,
$$

for any fixed $p_{1} \neq p_{2}$, which agrees (for large $n$ ) with the assumption of independence of group numbers of fixed sizes, in the stochastic context of SE. In 13 it was also shown that for a wide class of the parameter functions $a$ and a fixed $p$,

$$
E K_{p} \sim k_{p}, \quad \text { as } n \rightarrow \infty,
$$

where $k_{p}$ is the equilibrium solution of the continuous version of SE. However, it was found that both (4.20) and (4.21) fail when the group size $p=p(n) \rightarrow \infty$, as $n \rightarrow \infty$. The latter leads to the crucial difference in the behavior of stochastic and deterministic solutions at equilibrium. It is plain that the difference between the two models is the consequence of the mass conservation law (4.1) that contradicts the assumption of independence.

In the paper 20] by G. Freiman and B. Granovsky, Khintchine's probabilistic method was brought to the scenario. With the help of this method, asymptotic formulae for the partition function for the invariant measure (4.4) were derived in the case when $a_{n} \sim n^{l-1}, l>0, n \rightarrow \infty$. In [20] one can also find a sketch of the history of Khintchine's method.

I. Jeon [27] found sufficient conditions on intensities of coagulation and fragmentation in SE under which the gelation phenomena occurs. Note that these conditions are not satisfied for the reversible intensities generated by the class $\mathcal{F}(l), l>0$, of parameter functions considered in the present paper.

The paper 32] by P. Laurencot and D. Wrzosek introduced a version of SE with coagulation and collisional fragmentation. The latter means that the fragmentation ( = breakage) occurs only as a result of a collision of two clusters.

Essentially, all stochastic and deterministic processes discussed so far are meanfield models, in the sense that the rates of coagulation and fragmentation depend on the sizes of interacting groups only. J. R. Norris 35 formulated a continuum version of SE in the case when the coagulation rates depend not only on the particle masses but also on some other characteristics of the clusters (e.g., the shape of the cluster, the types of basic particles that form the cluster, etc.).

\section{Application 2: Random combinatorial structures (RCS)}

A combinatorial structure (CS) of a size $n$ is defined as a union of components (= nondecomposable elements) of sizes $1,2, \ldots, n$, and by RCS we mean the uniform probability distribution on the finite set of all $p_{n}$ CS's of size $n$. The RCS induces the component size counting process $\mathbf{K}^{(n)}=\left(K_{1}^{(n)}, \ldots, K_{n}^{(n)}\right)$, where $K_{i}=K_{i}^{(n)}, i=1,2 \ldots, n$, are the numbers of components (in a randomly chosen CS) of sizes $i=1,2, \ldots, n$, subject to the total mass conservation law (4.1). It was long ago understood that for a wide class of RCS's the distribution laws $\mathcal{L}$ 
of the processes $\mathbf{K}^{(n)}$ have the following common feature called the conditioning relation (for references see the monograph [4], Ch. 2, by R. Arratia, A. Barbour, and S. Tavaré and [5], by R. Arratia and S. Tavaré):

$$
\mathcal{L}\left(\mathbf{K}^{(n)}\right)=\mathcal{L}\left(Z_{1}, \ldots, Z_{n} \mid \sum_{i=1}^{n} i Z_{i}=n\right), \quad n=1,2, \ldots,
$$

where $Z_{1}, Z_{2}, \ldots$ are independent integer valued random variables. The great importance of the conditioning relation (5.22) is based on the following two interrelated facts that hold for a variety of instances of RCS's.

- The distribution of $Z_{i}, i=1,2, \ldots$, is of one of the following three types:

(i) $\operatorname{Poisson}\left(\frac{m_{i} x^{i}}{i !}, x>0\right)$, (ii) Negative binomial $\left(m_{i}, x^{i}, x \in(0,1)\right)$

or (iii) Binomial $\left(m_{i}, \frac{x^{i}}{1+x^{i}}, x>0\right)$, where in all the cases $x$ is a free parameter and $m_{i}$ is the number of components of size $i$.

- Corresponding to the type of the distribution of $Z_{i}$, the relationship between the two key sequences $\left\{p_{n}\right\}$ and $\left\{m_{i}\right\}$ has the form:

(i)

$$
\sum_{n=0}^{\infty} \frac{p_{n} z^{n}}{n !}=\exp \left(\sum_{i=1}^{\infty} \frac{m_{i} z^{i}}{i !}\right)
$$

$$
\sum_{n=0}^{\infty} p_{n} z^{n}=\prod_{i=1}^{\infty}\left(1-z^{i}\right)^{-m_{i}}
$$

$$
\sum_{n=0}^{\infty} p_{n} z^{n}=\prod_{i=1}^{\infty}\left(1+z^{i}\right)^{m_{i}}
$$

In accordance with the above, the following three basic classes of CS's are distinguished (4]): (i) assemblies, (ii) multisets and (iii) selections.

First, we immediately see from (5.23) that assemblies are incorporated into our setting (2.10) with $a_{n}, c_{n}$ having a clear combinatorial context: $a_{n}=\frac{m_{n}}{n !}, \quad c_{n}=$ $\frac{p_{n}}{n !}, \quad n=1,2, \ldots$

A quite different approach leading to the relationship (5.23) is widely known in combinatorics (for references see [39], Ch. 5). In this field, (5.23) which is called the exponential formula, expresses the general enumerative principle for posets, that may be regarded as disjoint unions of their connected components. In particular,

$$
S(z)=\sum_{n=1}^{\infty} \frac{m_{n} z^{n}}{n !} \quad \text { and } \quad g(z)=\sum_{n=0}^{\infty} \frac{p_{n} z^{n}}{n !}
$$

are called exponential generating functions for the number of connected components and for the total number of posets, respectively. Note that in the graph theory, (5.23) is deduced from the generalized scheme of allocation (see [31], Ch. 1, by V. Kolchin), the latter being equivalent, in effect, to the aforementioned enumerative principle.

Multisets can be also put into the framework of (2.10), by exponentiation of the generating function for the sequence $\left\{m_{i}\right\}$ (for references see Ch. 2 of the monograph [11], by S. Burris). 
We write

$$
\prod_{i=1}^{\infty}\left(1-z^{i}\right)^{-m_{i}}=\exp \left(\sum_{i=1}^{\infty} m_{i} \log \left(1-z^{i}\right)^{-1}\right)=\exp \left(\sum_{n=1}^{\infty} z^{n} \sum_{j, k: j k=n} \frac{m_{j}}{k}\right)
$$

to get from (5.24), $c_{n}=p_{n}, \quad a_{n}=\sum_{j, k: j k=n} \frac{m_{j}}{k}$.

Thus, the counting processes $\mathbf{K}^{(n)}$ for assemblies and multisets satisfy

$$
\mathcal{L}\left(\mathbf{K}^{(n)}\right)=\mu_{n},
$$

where $\mu_{n}$ is the measure given by (4.4) with $\gamma=1$ and the parametric function $a$ is as indicated above.

Although, in the case of multisets, $a_{n}$ lacks a combinatorial meaning, it turns out that, under a certain condition, the asymptotic behaviors of the two sequences $\left\{a_{n}\right\}$ and $\left\{m_{j}\right\}$ are similar.

Proposition 3 ([8], Lemma 5.1). If the sequence $\left\{m_{j}\right\}$ in (5.26) is such that

$$
\lim _{j \rightarrow \infty} \frac{m_{j}}{m_{j+1}}=h, \quad 0<h<1,
$$

then $a_{j} \sim m_{j}, \quad n \rightarrow \infty$.

By virtue of Remark 5 this means that our results on clustering are applicable for multisets with $m_{j} \sim h^{j} j^{l-1} L(j), j \rightarrow \infty, \quad l, h>0$. Now notice that applying the exponentiation procedure in the case of selections we arrive at an alternating sequence $\left\{a_{n}\right\}$. This says that this case is beyond the scope of the setting of the present paper.

The asymptotic behavior of counting processes was fully explored for the subclass of RCS's characterized by the logarithmic condition,

$$
\lim _{i \rightarrow \infty} i P\left(Z_{i}=1\right)=\lim _{i \rightarrow \infty} i E Z_{i}=\theta,
$$

for some $\theta>0$, where the random variables $Z_{i}, \quad i=1,2, \ldots$, are as in (5.22). Such RCS's are called logarithmic. A comprehensive exposition of the research for this case is given in 4 .

The classical example of a logarithmic RCS is the seminal Ewens sampling formula (ESF) given by $a_{n}=\frac{\theta}{n}, n \geq 1, \theta>0$. It originated in population genetics (1972) and was extensively investigated by many authors in relation to a variety of models. In particular, it was proved that the normalized ESF converges weakly to the Poisson-Dirichlet law (see [16, 25] and references therein). The counting process induced by ESF can be interpreted as a theta-biased random permutation (44, Ch. 3). The theory of the limiting behavior of the counting process in the case $\theta=1$ (= random permutations) was shaped by V. L. Goncharov (1942), L. A. Shepp and S. P. Lloyd (1966) and A. M. Vershik and A. A. Shmidt (1977) (for references see [4], Ch. 1 and 31, Ch. 4).

On the other hand, integer partitions provide an example of a nonlogarithmic RCS. Partitions can be formally defined as a multiset with $m_{i}=1, \quad i \geq 1$. Thus, (5.26) gives for this case

$$
a_{n}=\sum_{d \in D_{n}} \frac{1}{d} \leq \sum_{j=1}^{\frac{n}{2}} \frac{1}{j},
$$

where $D_{n}$ is the set of all divisors of $n$. Consequently,

$$
1 \leq a_{n} \leq \log n, \quad n \rightarrow \infty
$$


which indicates that the case of partitions can be approximated by the class of parameter functions $\mathcal{F}_{l}$ with $l=1$.

In the next section we explain that $q$-colored linear forests (treated as posets) is a RCS with $a \in \mathcal{F}_{1}$. In the conclusion, we make the following

Remark 8. The logarithmic condition (5.29) fails for the class $\mathcal{F}_{l}, l \neq 0$, of parameter functions $a$. On the other hand, the Lyapunov condition, and consequently the normal local limit theorem, hold only when $l>0$. This explains why in the study of the clustering problem, the cases $l=0, l>0$ and $l<0$ should be distinguished, with basically different asymptotic tools being employed. The third case that includes such RCS's, as forests of labelled (unlabelled) trees, was recently explored in [6], by A. Barbour and B. Granovsky. Correspondingly, three very different pictures of clustering were discovered. A specific feature of clustering in the case $l>0$ considered in the present paper is the existence of a threshold value for the size of the maximal component (cluster). So, this appears to be the only case (among $a \in \mathcal{F}_{l}$ ) in which the gelation phenomena is not seen.

In the context of RCS's, the aforementioned principle of asymptotic independence of small groups (= components) has been widely discussed for a long time, in connection with the conditioning relation (5.22). The independence was proved for the logarithmic RCS's (4, Ch. 4) and in the case $E Z_{j}=j^{l-1} L(j), l \leq 0$, in [6].

\section{Application 3: Additive number systems (ANS)}

ANS's provide a very general setting that encompasses multisets, as defined in the previous section. Following 11] by S. Burris, an ANS $\mathcal{A}$ is a countable free commutative monoid $A=\{v\}$ with a given set $P$ of nondecomposable elements (= generators) and with an additive norm $\|\bullet\|$, such that the set

$$
\{v \in A:\|v\|=n\}
$$

is finite for all $n \in \mathcal{N}$. This definition implies that each $v \in A$ is a sum (= union) of elements of $P$. Denoting $c_{n}, p_{n}$ the number of elements in $A$ and $P$ correspondingly with norm $n$, an enumerative argument yields the following characteristic identity for ANS's:

$$
\sum_{n \geq 0} c_{n} x^{n}=\prod_{n \geq 1}\left(1-x^{n}\right)^{-p_{n}}, \quad 0 \leq x \leq \rho<1 .
$$

By the exponentiation of the RHS of (6.30), we get the alternative version of the above identity:

$$
g(x)=\exp \left(\sum_{m \geq 1} \frac{P\left(x^{m}\right)}{m}\right), \quad 0 \leq x \leq \rho<1,
$$

where $g$ and $P$ are the generating functions for the sequences $\left\{c_{n}\right\}$ and $\left\{p_{n}\right\}$ respectively:

$$
g(x)=\sum_{n \geq 0} c_{n} x^{n}, \quad P(x)=\sum_{n \geq 0} p_{n} x^{n}, \quad 0 \leq x \leq \rho<1 .
$$

Now (6.31) can be rewritten as (2.10) with

$$
a_{n}=\sum_{j m=n} \frac{p_{j}}{m} .
$$


As we already mentioned before, the sequence $\left\{a_{n}\right\}$ defined by (6.33) usually does not exhibit a regular asymptotic behaviour, i.e., does not belong to the class $\mathcal{F}(l), l>0$.

On the other hand, ANS's with $p_{n}$ satisfying the condition of Proposition 3 are a nice exception to the above phenomenon. An example of such a structure is the set of $q$-colored linear forests, treated as posets, in which case $p_{n}=q^{n}$ (see [11], p. $24)$. It is also important to note that the radius of convergence of the generating series in (6.32) cannot be greater than 1 .

We wish now to demonstrate that the known asymptotic result on ANS's that facilitated the development of Compton's density theory is a particular case of our asymptotic formula for $c_{n}$. In 1992 it was proven by J. Knopfmacher, A. Knopfmacher and R. Warlimont (see for references [11, Theorem 5.17, p. 94) that if in (6.30),

$$
p_{n}=h q^{n}+O\left(q_{1}^{n}\right), \quad h>0, \quad q>1, \quad 0<q_{1}<q,
$$

then

$$
c_{n} \sim h_{1} q^{n} \frac{e^{2 \sqrt{h n}}}{n^{\frac{3}{4}}}, \quad h_{1}>0, \quad n \rightarrow \infty,
$$

where $h_{1}>0$ is a constant which was not specified. This result was obtained with the help of complex analysis.

By virtue of Proposition 3, we see that $a_{n} \sim p_{n}, n \rightarrow \infty$, which together with Remark 5, permits us to apply our asymptotic formula (3.66) with $l=1$ and $L(n) \equiv h$. So, in the case considered, $L^{*}(n) \equiv h^{-1}$ and we have in (3.30), $L_{1}(n)=h^{-1 / 2}$. Consequently, by the asymptotic formulae in Section 3, we have $\underline{\sigma}_{n}^{(n)} \sim n^{-1 / 2} h^{1 / 2}$, $\underline{S}_{n}^{(n)}\left(e^{-\underline{\sigma}_{n}^{(n)}}\right) \sim h^{1 / 2} n^{1 / 2}-h / 2+O\left(\underline{\sigma}_{n}^{(n)}\right)$, and $\left(\underline{B}_{n}^{n}\right)^{2} \sim 2 h^{-1 / 2} n^{3 / 2}$, as $n \rightarrow \infty$. Substituting this in (3.66), recovers (6.34), while specifying $h_{1}=(2 \sqrt{\pi})^{-1} h^{1 / 4} e^{-h / 2}$.

The central problem in the theory of ANS's is the study of the asymptotic density $\delta(B)$ of a subset $B$ of a monoid $A$ :

$$
\delta(B)=\lim _{n \rightarrow \infty} \frac{b_{n}}{c_{n}},
$$

where $b_{n}$ is the number of elements of $B$ with norm $n$.

It follows from (6.33) that the quantities $\underline{d}_{n}^{(r)}, \bar{d}_{n}^{(r)}$ in the clustering problem considered in the present paper can be regarded as the densities of the subsets, say $B_{1}, B_{2} \subseteq A$, such that the maximal (minimal) norm of generators of elements of $B_{1}\left(B_{2}\right)$ satisfies a certain condition.

The fundamental result in this field is Compton's density theorem (1989) (see 11], Ch. 4, 5) that establishes sufficient conditions for existence of an asymptotic density of all partition sets of an ANS $\mathcal{A}$.

Coming back to the example of $q$-colored linear forests (as posets), our Theorem 2, applied with $l=1$ and $L(n) \equiv h$, gives the asymptotic density of the aforementioned sets $B_{1}, B_{2}$.

\section{ACKNOWLEDGEMENT}

The research of the second author was supported by the Fund for the Promotion of Research at Technion.

The referee's criticism and remarks contributed to the improvement of the exposition of the paper. 


\section{REFERENCES}

[1] M. Aizenman and T. A. Bak, Convergence to equilibrium in a system of reacting polymers. Commun. Math. Phys. 65 (1979), 203-230. MR80d:80008

[2] D. J. Aldous, Deterministic and stochastic models for coalescence (aggregation, coagulation): a review of the mean-field theory for probabilists. Bernoulli 5 (1999), 3-48. MR 2001c:60153

[3] D. J. Aldous, Emergence of the giant component in special Marcus-Lushnikov processes. Random Structures Algorithms, 12 (1998), 179-196. MR.99g:60128

[4] R. Arratia, A. Barbour, S. Tavaré, Logarithmic combinatorial structures: a probabilistic approach, EMS Monographs in Math., European Math. Soc., Zürich, 2003. MR2032426

[5] R. Arratia, S. Tavaré, Independent process approximations for random combinatorial structures. Adv. Math. 104 (1994), 90-154. MR95c:60010

[6] A. D. Barbour, B. Granovsky, Random combinatorial structures: the convergent case. Preprint, math.Pr/0305031, math@arXiv.org. (2003).

[7] J. Bell, Sufficient conditions for zero-one laws. Trans. Amer. Math. Soc. 354 (2002), 613-630. MR 2002j:60057

[8] J. Bell, S. Burris, Asymptotics for logical limit laws: When the growth of the components is in RT class. Trans. Amer. Math. Soc. 355 (2003), 3777-3794. MR2004h:03072

[9] J. Bertoin, Homogeneous fragmentation processes. Probab. Theory Relat. Fields 121 (2001), 301-318. MF 2002j:60127

[10] N. H. Bingham, C. M. Goldie, J. L. Teugels. Regular variation. Encyclopedia of Mathematics and its Applications 27, Cambridge University Press, 1987. MR88i:26004

[11] S. Burris, Number theoretic density and logical limit laws. Mathematical surveys and monographs 86, American Mathematical Society, Providence, RI, 2001. MR2002c:03060

[12] J. Deshouillers, G. Freiman, W. Moran, On series of discrete random variables,1: real trinomial ditributions with fixed probabilities. Asterisque 258 (1999), 411-423. MR2000j:60028

[13] R. Durrett, B. Granovsky, S. Gueron, The equilibrium behaviour of reversible coagulationfragmentation processes. J. of Theoretical Probability 12 (1999), 447-474. MF 2000g:82013

[14] A. Eibeck, W. Wagner, Stochastic particle approximations for Smoluchowski's coagulation equation. Annals of Applied Probab. 11 (2001), 1137-1165. MR.2002k:60208

[15] S. Evans, J. Pitman, Construction of Markovian coalescents. Ann. Inst. Henri Poincaré 34 (1998), 339-383. MR 99k:60184

[16] W. J. Ewens, Remarks on the law of succession. Athens conference on applied probability and time series analysis, v. 1, 229-244, 1995, Lecture Notes in Statistics, 114, Springer, NY, 1996. MR $98 \mathrm{~g}: 92001$

[17] H. Eyring, D. Henderson, B. J. Stover, E. M. Eyring, Statistical mechanics and dynamics. NY, 1964

[18] W. Feller, An introduction to probability theory and its applications, v. II, Wiley, NY, 1966. MR 35:1048

[19] G. Freiman, Waring's problem with an increasing number of terms. Elabuz. Goz. Ped. Inst. Učen. Zap. 3, 105-119, 1958 (in Russian). MR.41:166

[20] G. Freiman, B. Granovsky, Asymptotic formula for a partition function of reversible coagulation-fragmentation processes. J. Isr. Math., 130 (2002), 259-279. MR 2003i:60036

[21] G. Freiman, J. Pitman, Partitions into distinct large parts. J. Austral. Math. Soc. (Series A) 57 (1994), 386-416. MR95h:11110

[22] G. Freiman, A. Vershik, Yu. Yakubovitz, A local limit theorem for random strict partitions. Th. Probab. Appl. 44 (2000), 453-468. MR2002c:11134

[23] B. V. Gnedenko, A. N. Kolmogorov, Limit distributions for sums of independent random variables. Addison-Wesley, 1954. MR16:52d

[24] S. Gueron, The steady-state distributions of coagulation-fragmentation processes. J. Math. Biol. 1 (1998), 1-27. MR 99g:82055

[25] U. Hirth, A Poisson approximation for the Dirichlet law, the Ewens sampling formula and the Griffith-Engen-McCloskey law by the Stein-Chen coupling method. Bernoulli 3 1997, 225-232. MR $98 \mathrm{~m}: 60026$

[26] I. A. Ibragimov, Yu. V. Linnik, Independent and stationary sequences of random variables. Walters-Noordhoff, Groningen, 1971. MR 48:1287

[27] I. Jeon, Existence of gelling solutions for coagulation-fragmentation equations. Commun. Math. Phys., 194 (1998), 541-567. MF 99g:82056 
[28] I. Jeon, P. March, B. Pittel, Size of the largest cluster under zero-range invariant measures. Ann. Probab. 28 (2000), 1162-1194. MR 2002j:60183

[29] A. I. Khinchin, Mathematical foundations of quantum statistics. Graylock Press, Albany, N.Y., 1960. MR22:2081

[30] F. Kelly, Reversibility and stochastic networks. Wiley, 1979. MR81j:60105

[31] V. Kolchin, Random graphs. Encyclopedia of Mathematics and its Applications, 53, Cambridge Univ.Press, 1999. MR2001h:60015

[32] P. Laurencot, D. Wrzosek, The discrete coagulation equations with collisional breakage. J. Stat. Phys. 104 (2001), 193-220. MR2002k:82056

[33] L. Mutafchiev, Local limit theorems for sums of power series distributed random variables and for the number of components in labelled relational structures. Random structures and Algorithms, 3 (1992), 404-426. MR.93k:60060

[34] J. Norris, Smoluchowski's coagulation equation: uniqueness, non-uniqueness and a hydrodynamic limit for the stochastic coalescent. Ann. Appl. Probab. 9 (1999), 78-109. MR2000d:82034

[35] J. Norris, Cluster coagulation. Comm. Math. Phys., 209 (2000), 407-435. MR2002c:82079

[36] A. G. Postnikov, Introduction to analytic number theory. Translations of Mathematical Monographs, 68, AMS, 1987. MR89a:11001

[37] E. Seneta, Functions of regular variation. Lecture Notes in Mathematics, 506, Springer, NY, 1976. MR 56:12189

[38] M. V. Smoluchowski, Versuch einer mathematischen Theorie der Koagulationskinetik kolloider Lösungen. Z. Phys. Chem. 92 (1917), 129-168.

[39] R. P. Stanley, Enumerative combinatorics, Volume 2. Cambridge University Press, 1999. MR 2000k:05026

[40] N. Wax, ed., Selected papers on noise and stochastic processes. Dover, 1954. MR.15:970a

[41] P. Whittle, Statistical processes of aggregation and polymerization. Proc. Camb. Phil. Soc., 61 (1965), 475-495. MR 31:3151

[42] P. Whittle, Systems in stochastic equilibrium. Wiley, 1986. MR88c:60162

School of Mathematical Sciences, Raymond and Beverly Sackler Faculty of Exact Sciences, Tel-Aviv University, Ramat-Aviv, Tel-Aviv, Israel

E-mail address: grisha@math.tau.ac.il

Department of Mathematics, Technion-Israel Institute of Technology, Haifa, 32000 , ISRAEL

E-mail address: mar18aa@techunix.technion.ac.il 